\title{
An Ontology-Based Collaborative Framework for Decision Support in Engineering
}

\author{
Hauke Arndt, Rüdiger Klein \\ DaimlerChrysler Research Department, Knowledge Based Engineering Group
}

\begin{abstract}
In this paper the MOKA framework as an expressive and generic model of design problem solving is extended by a model of design rationale. The MOKA framework is based on a product and process model which allows an explicit description of requirements, design descriptions, problem solving steps, decisions, etc. In this paper it is shown how decisions and their rationale can be integrated into this explicit product and process description. This tight conceptual integration of design decisions and their rationale into the MOKA framework is achieved by specifying a design rationale ontology which fits into the MOKA framework.
\end{abstract}

Key words: Product-Model, Process-Model, Design Rationale, Decision Support, MOKAFramework, Ontology of Design Rationale

\section{INTRODUCTION}

A typical technical product today tends to be very complex, and so does the corresponding design and engineering process. Information systems of any kind are ubiquitous now in engineering in order to manage this complexity. Though in the meanwhile many of them (like CAD and PDM systems) reached a considerable maturity, there are challenging problems left. One of them is interoperation: though very important for collaboration in engineering, the various IT systems follow different approaches to information modeling and problem solving. This makes their communication and collaboration complicated and error prone. Another such challenging problem is adequate IT support for decision making (by systems or humans). Both issues are closely related to each other: interoperation is not primarily a data exchange problem - at most it is an issue of collaborative problem 
solving, i.e. of coordinated decision making. And decision making is one of the main elements in engineering problem solving - with many relations to practically all aspects of the collaborative engineering process. Both aspects - interoperation and decision support - gain special importance in the Internet/intranet context. Using these new technologies to their full extend and benefit requires - among others - a more sophisticated approach to interoperation and decision support.

IT support of decision making is by far not a new problem. There is a number of approaches describing design rationale (see for example [Blessing1994; M.Klein1992; MacLean1996; Shum1996]). Although these approaches are quite expressive they are of limited use, because in most cases the rationale is described separated from the information about the product and the design process. Currently, there is no satisfying practical approach [Lei et al. 1995; Brazeir et al. 1995; Gero 1998]. This is quite natural taking the complexity of product and process knowledge into account - as well as the many different aspects of design decisions and their rationale.

In this paper we describe an integrated approach to design rationale bringing together an explicit model of design and a sophisticated model of design rationale. We use the MOKA framework [Klein2000] as an expressive and well-founded model of design and an adaptation of the QOC design rationale model [MacLean1996]. Combining both will result in an expressive and usable model of design and design rationale.

In this paper we describe first the MOKA framework [Klein2000] (chap. 2) and important aspects of the design process (chap. 3). In chap. 4 an ontology-based model for representing the process- and product-model of the MOKA framework is presented. These chapters build the basis for chap. 5 where the MOKA framework is extended in order to integrate design rationale. In chapter 6 we present an example for the design rationale framework. Chap. 7 contains a summary and our conclusions.

\section{A FRAMEWORK FOR DESIGN DECISION MAKING: THE MOKA FRAMEWORK}

In this paper we describe a model of decision making in engineering and the rationale behind it. It is based on and integrated into the MOKA framework [Klein2000]. As a pre-requisite for modeling decisions and their rationale, this framework allows us to capture the complexity of product and process knowledge and the interrelation between them (see figure 1).

The MOKA framework comprises an expressive product model which allows us to represent the whole complexity of product information in a clear, well structured and formalized way. Especially, not only the 
completed design can be modeled but also all related and intermediate product information like generic knowledge, design requirements of any kind, function-structure relations, behaviors, etc.

In the MOKA framework, this product model is closely related to a process model. This is essential because design problems can normally not be solved in a pre-defined manner - they need complex processes which are essentially determined by the product to be designed. Consequently, engineering problem solving is an incremental, interactive, and mostly hierarchical decision process with propagation of decision consequences.

\section{THE DESIGN PROCESS}

In order to get a better understanding of what design decisions are and what their rationale can look like we need a more detailed description of the design process:

Typically, the design process starts with a set of initial requirements ${ }^{1}$ which may be incomplete and even contradictory. It ends with a complete design description which fulfills the (adjusted) requirements and is consistent with the general design and engineering knowledge. This process transforms the initial state into the final result. It is a kind of complex dynamic search based on a continuous interplay of synthetic and analytical steps.

Synthetic steps elaborate the design description, add more and more information to it in order to fulfill requirements and make it complete. Analysis steps are used to investigate the various relations between design and requirements, among conflicting requirements, etc. which may result in relaxed or new requirements. This is essential because a key property of design problem solving is its non-monotonic nature: adding new information to a design description may change other properties (for instance, behavior, geometric shape, or total weight).

Search is needed in order to escape from conflicts, dead ends, or inappropriate design states. Inappropriate are those states which fulfill all "hard" requirements and constraints but are non-optimal with respect to some "soft" requirements. Optimality is important in many design problems: frequently we are not only interested to find any solution but one which is optimal (or at least close to optimal) with respect to some criteria.

Due to the complexity of products and processes in design the corresponding search spaces tend to be huge. In practice, there is frequently no explicit description of all possible alternatives in a given state of design

${ }^{1}$ Leaving aside case adaptations or variational design which can be "reduced" somehow to the basic case. 
problem solving. The human designer takes heuristic knowledge in order to focus on those problem solving steps which look promising - leaving other alternatives unattended.

Currently, it seems not to be realistic to have an automated design system: engineering knowledge is partially so complicated that it can not be modeled adequately with current means. So, essential parts of relevant knowledge have to be left with the human user. The system only has parts of the knowledge available. Typically, a human designer will use such a design support system as an intelligent assistant. This system should propagate consequences from decision through the whole product and process model, it should analyze conflicts, make proposals to the human about how to proceed, etc. The integration of design rationale into this process is an essential pre-requisite for the practicability of collaborative problem solving in design.

\section{AN ONTOLOGY-BASED MODEL OF PRODUCT AND PROCESS KNOWLEDGE}

This all together forms a complex set of requirements to information modeling. In order to fulfill them, the MOKA framework allows us to structure this complex information by product and process ontologies [Gruber1993]. An ontology is a set of interrelated formal definitions of all notions relevant in a certain domain. This allows us to describe explicitly the main information categories and their relations in a domain - an important issue for the integration of different information models.

In the MOKA framework we have the following information categories (see UML diagram in figure 1):

- a meta model (upper structure) as the core of the ontology [Klein2000].

This allows us to capture the main knowledge categories and their relations, and to structure the whole bunch of product and process knowledge accordingly;

- following this upper structure, the product model (product description in fig. 1) consists of generic as well as concrete information about functions, structures, and behaviors using all kinds of classes, attributes, relations, hierarchies, constraints, and different views. Requirements and design descriptions are related to each other in a logical way, as they are to the generic design knowledge;

- the process model (process object) is described by synthesis and analysis tasks to elaborate requirements and design descriptions [Klein2000];

- the process model is connected to the product level by dynamic knowledge categories. They reflect the logical relations on the product 
level: for instance, fulfilled, unfulfilled or conflicting requirements, etc. (not all relations are shown in figure 1).

A strategic layer allows us to represent explicitly knowledge about the way decisions should be made: which criteria are more important then others, etc.

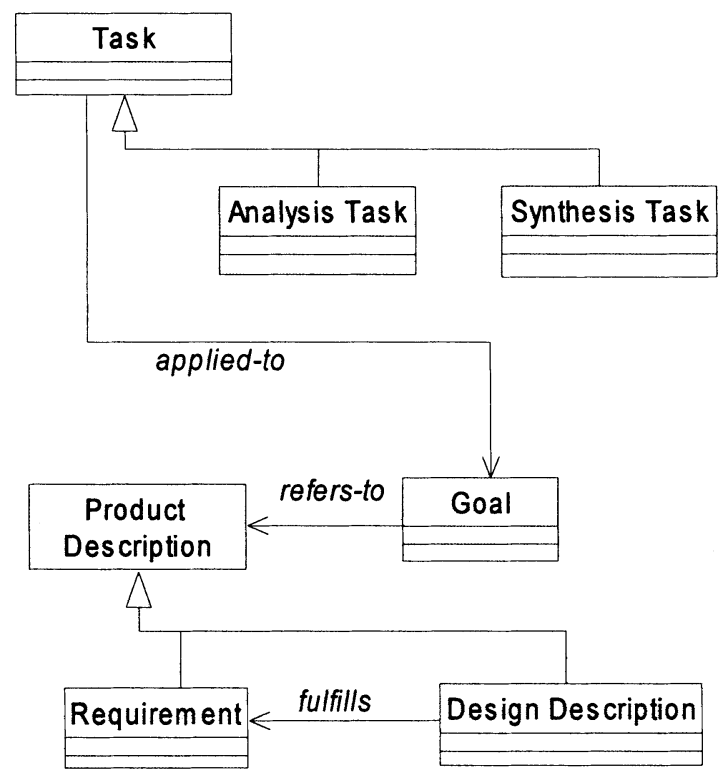

Figure 1. Simplified Product and Process Model of the MOKA framework (UML notation)

\section{AN ONTOLOGY FOR DECISIONS AND THEIR RATIONALE}

\subsection{A General Model of Design Rationale}

Currently, there are several models of decisions and their accompanying rationale (for example in [Blessing1994; M.Klein1992; MacLean1996; Shum1996; Chung1994]). Our approach is primarily based on QOC [MacLean1996] because it is self explaining as well as sufficiently expressive.

QOC uses the following information categories (ontology) for the description of design rationale: problem, criteria, alternative, decision and argument (see figure 2). Each category is specified in more detail by several 
attributes, e.g. a name, a textual description, references, remarks, etc. The problem category describes the 'what' of the decision to be made. Criteria are used to model the way in which decisions are made. Each criterion has an individual importance - a kind of weight - with respect to a given problem. These weights are essential for an appropriate decision making. Alternatives describe the several different solutions possible for the actual problem. The alternatives can either be technical solutions (product oriented alternatives) or distinct tasks of the problem solving process (process oriented alternatives). The decision describes the chosen alternative for a certain problem. An argument may (or may not) provide support to a certain alternative with respect to a relevant criterion.

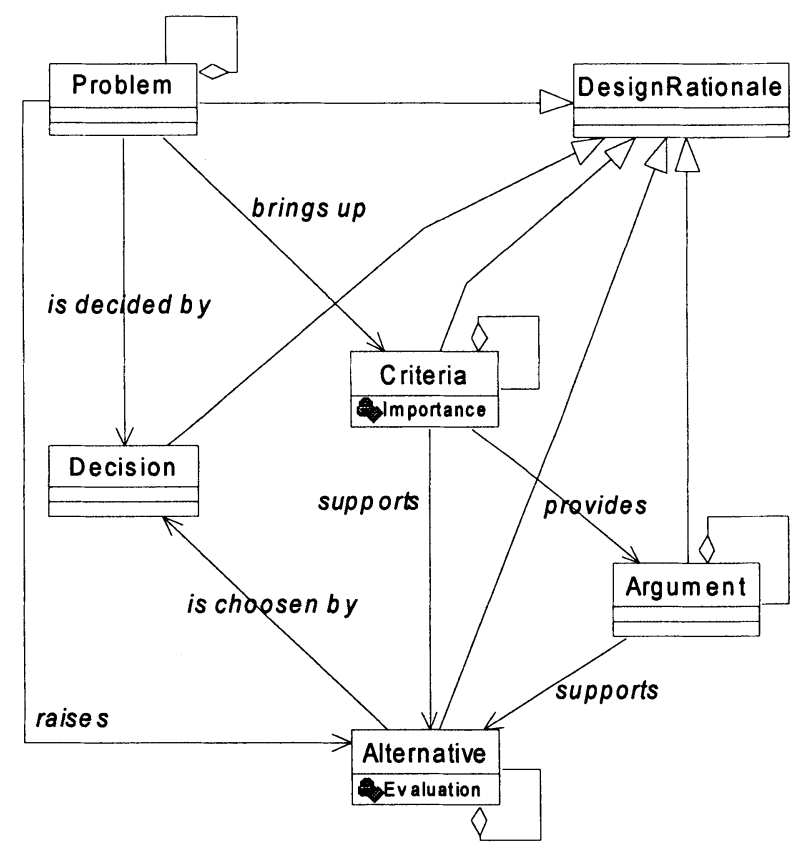

Figure 2. General Model of Design Rationale (UML-like notation)

In figure 2 also the relations between the several information categories of our QOC ontology are shown. All categories are related to each other, and most of them have additionally a breakdown structure (consists of, consists in - not shown here).

As mentioned earlier, an essential aspect of design rationale are their dependencies. Decisions and their rationale can depend on or conflict with 
each other. These dependencies can be modeled by two relations (affects and conflicts) on the problem category (also omitted here by space limitations).

\subsection{An Integrated Model of Products, Processes and their Design Rationale}

In order to support the understanding of a complex technical product, it is important to describe design rationale together within their context. In engineering, this is product and process related information - as modeled in the MOKA framework.

The MOKA framework can straightforwardly be extended such that the various kinds of decisions and their rationale can be modeled in an coherent way. Decisions can be characterized along four dimensions which build the core ontology of the decision and rationale information model:

1. The role within the design process (types of decisions): synthesis, analysis, conflict resolution, default, optimality decision, strategy of decision making;

2. The relation to product knowledge: to the involved requirements, functions, structures, design descriptions, hierarchy, etc.;

3. Dependencies: decisions are not independent from each other - one may be the pre-requisite of another one, a decision may be in conflict with other decisions, hierarchical decomposition of decision making ${ }^{2}$, etc.

4. Scope: in which context is a decision made, and how far should the consequences be considered.

So, we gained an ontology of decisions and their rationale integrated into the MOKA framework: a well-formalized, explicit, expressive and integrated approach. Well-formalized because it is based on a formal ontology of decisions and their rationale. Explicit, because all relevant relations to product, process and decision making are modeled in an explicit way. Expressive, because the whole expressiveness of the MOKA framework and the underlying ontology (explicit meta level, dynamic knowledge categories etc.) is available. And integrated, because decisions and their rationale are conceptually related to all other aspects of knowledge.

Figure 3 shows the ontology integrating our model of design rationale into the MOKA framework (describing both process and product model).

The MOKA framework describing the product and process model (see figure 1) on one side and the model of design rationale (see figure 2) on the other side are integrated by four relations. Tasks on the process level describe typical problems in the context of design rationale. This forms the

${ }^{2}$ Hierarchical decomposition of the problem and its problem solving process is a typical strategy in engineering. 
basis of a design rationale. Therefore the task is associated with alternatives (relation: raises), criteria (relation: brings up), and decision (relation: decides). All possible alternatives are product units (resp. requirement or design description). For this reason alternative is a super-class of product unit (all relations are inherited).

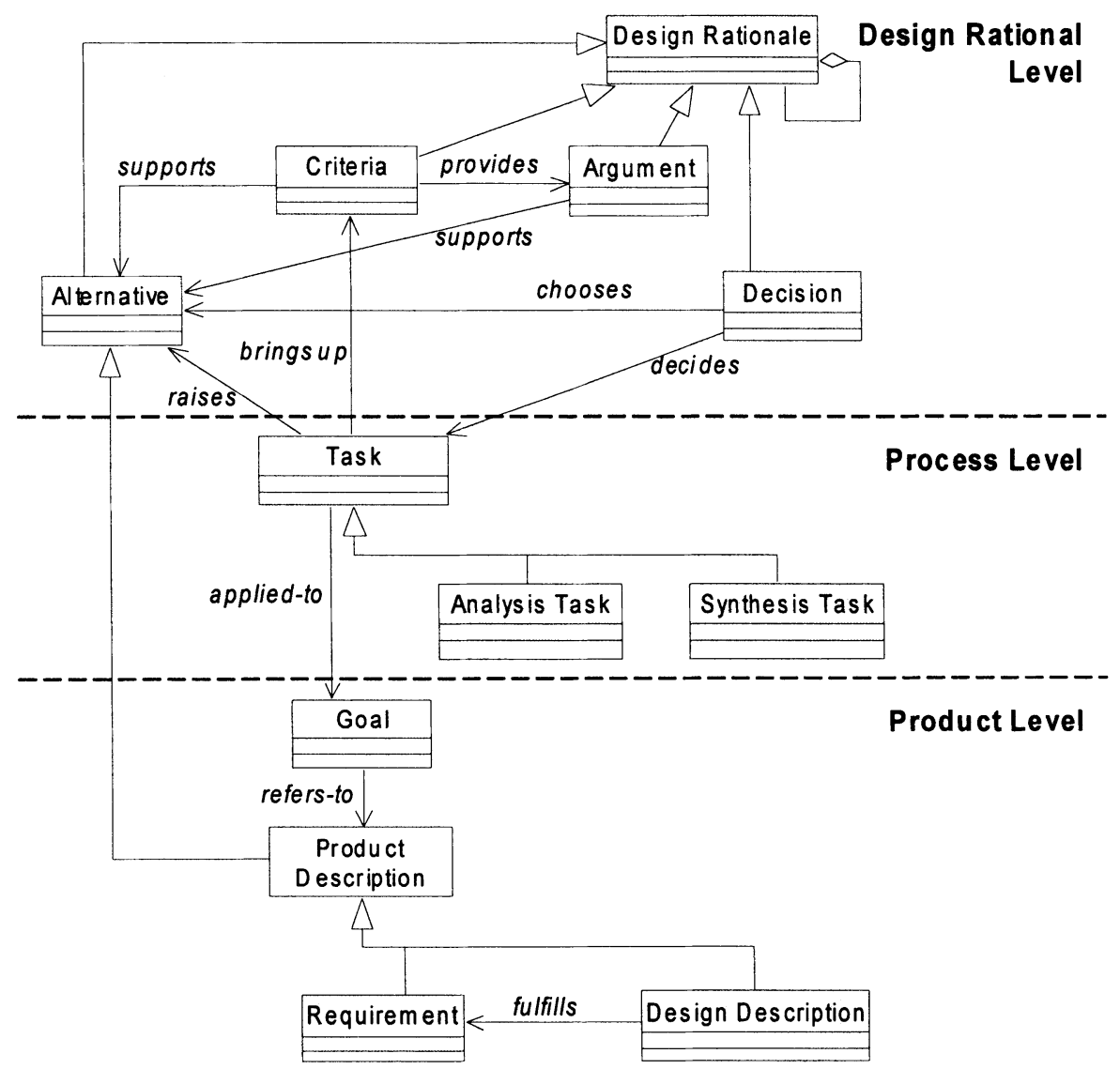

Figure 3. Model of Process, Product and Design Rationale (UML-like notation)

\subsection{How to use the information about decisions and their rationale}

The model presented here (see figure 3) allows us to describe the design product and its relation to the design process integrated with the underlying design rationale in their interdependencies. Also the scope of a design 
rationale is represented, as the knowledge about the process and the product is described in the same model.

This integrated well formalized approach allows us to use information about decisions and their rationale in the same way as other engineering information: it may be stored, retrieved, exchanged, etc. For instance, a query may be asked to a corresponding decision management system about all decisions currently influencing the length of a certain part $X$. The product relationship is obvious: length of part X; the process steps involved can be, for instance, default value assignment to the length of part $\mathrm{Y}$ and relation to length of $\mathrm{X}$ via a geometrical constraint; this also clarifies the dependency aspect; and the scope may be determined by the structural or functional hierarchy to which part $\mathrm{X}$ belongs. Alternatively, the decision management system could be queried for all decisions based on any geometrical default assignment.

\section{AN EXAMPLE: COOLANT PUMP}

Designing a coolant pump for vehicles is a typical engineering problem. It is a suitable example to be presented here as an illustration of our approach, because it is small enough to be understood easily but complex enough to demonstrate the usability of our approach.

In principle, a coolant pump (see figure 4) consists of spiral, impeller, bearing and a seal. The whole pump is located in a casing.

During the routine design process of a coolant pump a decision about the kind of the drive has to made. The drive of the pump could either be an electric, a belt (using a belt and a flange to transform mechanical energy from the vehicle's motor) or a direct drive. The decision about which drive to take is based mainly on cost aspects and on space and shape considerations of the pump housing.

In our approach this would be modeled as follows (see figure $5^{3}$ ): During the design process an analysis task "select" with the associated goal "kind of drive for coolant pump" is generated. This task forms also the basis for the design rationale. Associated to this goal are all possible design descriptions "electric drive", "belt drive", and "direct drive", which correspond to the possible alternatives on the design rationale level. The criteria needed to solve the actual problem properly are modeled with the help of the relation between design descriptions and decision arguments. With this knowledge arguments are generated which evaluate the alternatives with respect to the relevant criteria - providing the foundation for the decision to be made.

3 The whole information related to this application has been modeled in our ontology modeling tool OntoWorks (to be described in a forthcoming paper). 


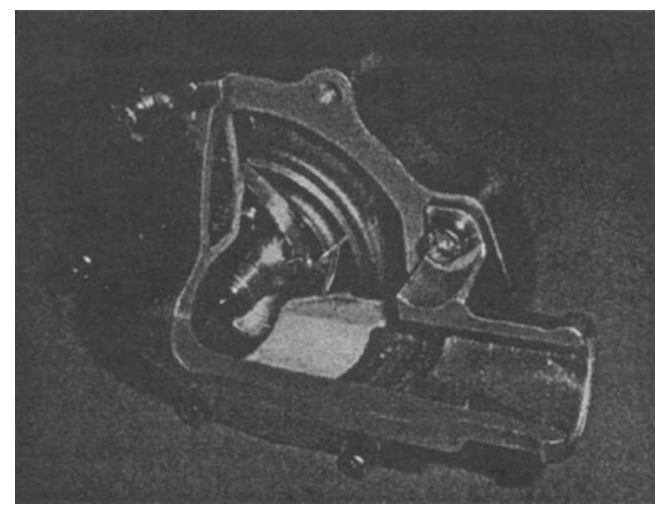

Figure 4. Picture of a Coolant Pump

In this way all decisions occurring during the design process can be described in an adequate way.

\section{SUMMARY AND CONCLUSIONS}

Design information tends to be quite complex: information about products, their requirements, behaviors, functions, properties, constraints, compositions, etc. - as well as information about problem solving: synthetic steps like instantiation of components or value assignments to properties and analytical steps to identify new requirements, find conflicts, etc. The MOKA framework provides expressive and well formalized means for the description of product and process related design information and the relations between them.

In this paper we presented an approach to integrate decision information and the accompanying rationale into this framework. Starting with an QOC like approach to model design decisions and their rationale, we suggested a way how these two models can be brought together. An ontology describing the MOKA framework and an ontology-based model of design decisions and the rationale behind them were integrated by defining the relations between information categories in both models. 


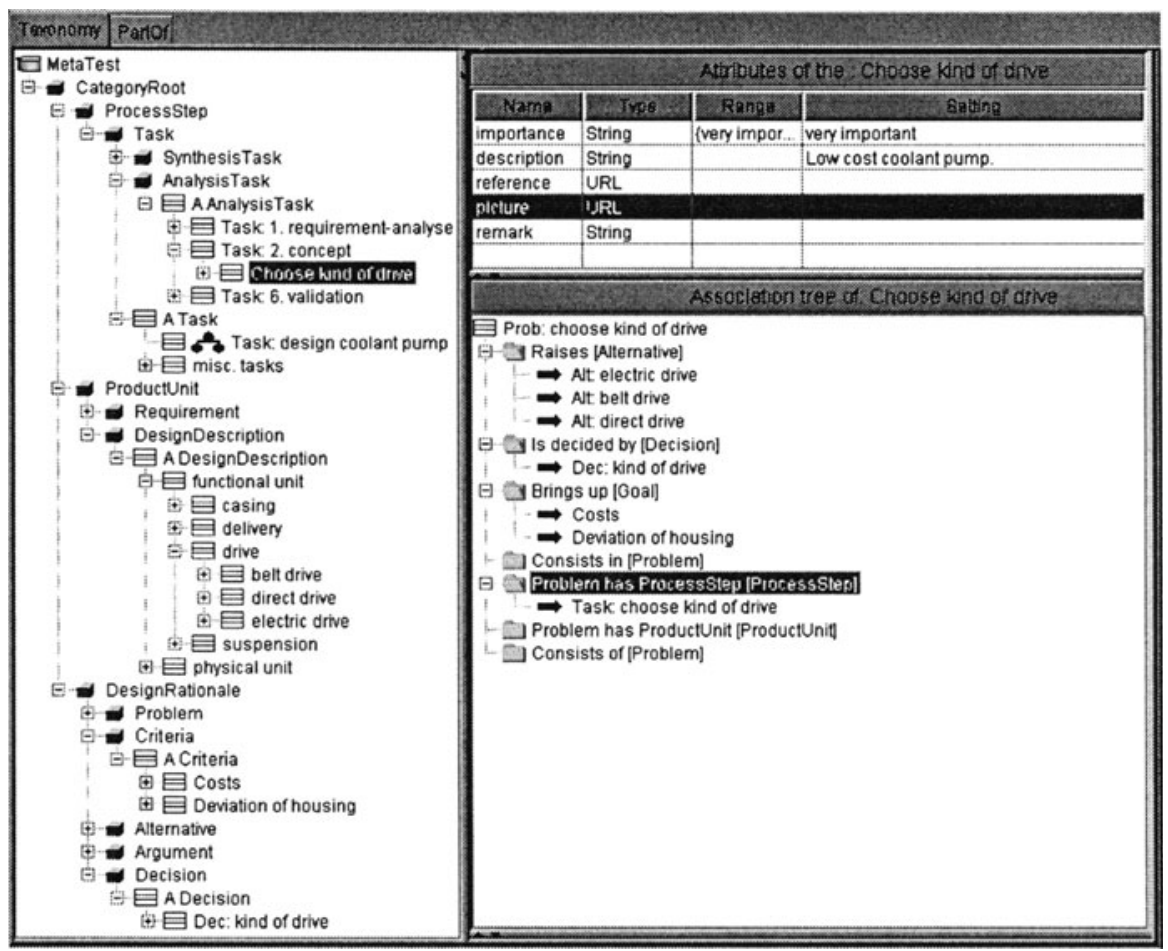

Figure 5. Modeling the Coolant Pump (Screenshot, not all details are actually shown)

This allows us to use information about decisions and their rationale in the same way as other engineering information: it may be stored, retrieved, exchanged, etc. With this explicit information about decisions and their rationale the collaboration in design problem solving can be supported much better then without it. Each partner (human or system) can use this information about the decisions made by others in the own decision process. This is essential for conflict resolution, optimality considerations, reuse of former cases, etc.

Of course, the approach presented here is only a first approximation. Its strength results from its tight conceptual integration into the MOKA framework as an explicit and well formalized model of design. Many aspects of design rationale - as used, for instance in teaching, explanation generation, or negotiations - are not fully covered here. A more sophisticated approach with more expressive models is under preparation. There we will focus especially on the integration and usage of background knowledge into more advanced applications of design rationale.

This gets special importance in an interconnected world. To use the Internet or intranet for product data exchange is one thing (important enough) - another, more important one is to use it for distributed 
collaborative problem solving. The exchange of complex information about the design process, about decisions and their rationale is indispensable for a distributed interconnected virtual enterprise. For this purpose, an explicit, expressive, and integrated model for decisions and their rationale is essential. Only this enables the partners really to exchange meaningful information and not just data. The Semantic $\mathrm{Web}$ will provide the necessary expressiveness and formalization [SemWeb].

\section{ACKNOWLEDGEMENTS}

We would like to thank Ute John, Michael Heinrich, Henrik Weimer, and the other members of the FT3/EW team for their useful comments on this paper.

\section{BIOGRAPHIES}

Hauke Arndt studied Mechanical Engineering and received his Diploma in 1999 from the Technical University of Berlin. Currently he's working on his Ph.D. thesis in the R\&T department of DaimlerChrysler. His main research interests are design rationale, engineering knowledge management and knowledge based systems.

Rüdiger Klein received a Diploma in Physics in 1975 with honors from Humboldt University in Berlin and a Ph.D. from the Academy of Sciences in Berlin in 1979. Since 1991 he has been a senior researcher in the R\&T department of the DaimlerChrysler AG, responsible for Knowledge Modeling research including ontologies, knowledge management, and knowledge based engineering.

From 1998 to 2000 Dr. Klein took part in the MOKA Esprit research project (No. 25418). Dr. Klein is a vice chair of the International AI in Design conference and a member of the program committee of the International ACM Symposium on Solid Modeling. He is the author and coauthor of more than 30 scientific publications.

\section{REFERENCES}

Brazier, F. M. T., van Langen, P. H. G., and Treur, J.: 1995, A logical theory of design, in J. S. Gero, and F. Sudweeks (eds), Preprints Advances in Formal Design Methods for CAD, Key Centre of Design Computing, University of Sydney, pp.247-271. 
Blessing, L. T. M.: 1994,. A process-based apporach to computer-supoorted engineering design, thesis university of twente, enschede, the netherlands, ISBN 0952350408.

Chung, P. W. H., Goodwin, R.: 1994, Representing Design History, in J. S. Gero and F. Sudweeks (ed.), Artificial Intelligence in Design '94, Kluwer Academic Publishers, Dordrecht, pp.735-752.

Gero, J. S.: 1998, Towards a model of designing which includes its situatedness, in H. Grabowski, S. Rude, and G. Grein (eds), Universal Design Theory, Shaker Verlag Aachen, pp.47-56.

Gruber, T.R.: 1993, A translation approach to portable ontology specifications, Knowledge Acquisition 5:199-200.

Klein, M.: 1992, DRCS: An integrated system for capture of designs and their rationale, in J. S. Gero (ed.), Artificial Intelligence in Design '92, Kluwer Academic Publishers, Dordrecht, pp.393-412.

Klein, R.: 2000, Knowledge modeling in design - the MOKA framework, in J. S. Gero (ed.), Artificial Intelligence in Design '00, Kluwer Academic Publishers, Dordrecht, pp.77-102.

Klein, R.: 2000, Towards an Integration of Engineering Knowledge Management and Knowledge Based Engineering. 2000 International CIRP Design Seminar: Design with Manufacturing: Intelligent Design Concepts Methods and Algorithms, May 16-18, 2000, Haifa, Israel.

Klein, R.: 1998, A Knowledge Level Theory of Design. G. Jagucci (ed.): Proceedings of the 10th International IFIP WG 5.2/5.3 Conference PROLAMAT 98, Trento, Italy, September 1998.

Lei, B., Taura, T., and Numata, J.: 1995, Representing the collaborative design process: $a$ product model-oriented approach, in J. S. Gero, and F. Sudweeks (eds), Preprints Advances in Formal Design Methods for CAD, Key Centre of Design Computing, University of Sydney, pp.274-291.

MacLean, A., et al: 1996, Question, options, and criteria: elements of design space analysis, in Moran, T.P., Carrol, J.M. (eds.), Design Rationale: Concepts, Techniques, and Use, Lawrence Erlbaum Associates, Publichers, Mahwah, New Jersey, 1996, ISBN 0-80581567-8

SemWeb: http://www.w3.org/2001/sw/

Shum, S. B. 1996: Analyzing the usability of a design rationale notation, in Moran, T.P., Carrol, J.M. (eds.), Design Rationale: Concepts, Techniques, and Use, Lawrence Erlbaum Associates, Publichers, Mahwah, New Jersey, 1996, ISBN 0-8058-1567-8

Takeda, H., Nishida, T.: 1994, Integration of aspects in design processes, in J. S. Gero and F. Sudweeks (ed.), Artificial Intelligence in Design '94, Kluwer Academic Publishers, Dordrecht, pp.309-326.

Protzen, J.-P., Harris, D., Cavallin, H.: 2000, Limited computation, unlimited design, in J. S. Gero (ed.), Artificial Intelligence in Design '00, Kluwer Academic Publishers, Dordrecht, pp.43-52. 